\title{
CRÉATIVITÉ ET RHÉTORIQUE DES DisCOURS PROMOTIONNELS SUR LES VINS NATURELS
}

\begin{abstract}
Audrey MoutaT ${ }^{1}$
Issus d'un engagement éthique des vignerons à l'égard de la terre et des consommateurs, les vins naturels résultent de pratiques œnologiques raisonnées réfutant toute méthode industrielle de viniculture et de vinification afin de (re)trouver l'expression naturelle du terroir et des qualités organoleptiques originelles.

Cette rupture avec la culture vitico-vinicole classique s'exprime à travers des stratégies communicationnelles transgressives qui ne s'enferment pas dans la norme de l'étiquette, la tradition des noms ou encore la canonicité du commentaire de dégustation classique.

Cet article vise ainsi à mettre au jour les dispositifs communicationnels engagés dans la promotion de ces vins atypiques.
\end{abstract}

Depuis quelques années, nous assistons à une médiatisation grandissante des vins sans (ou avec peu de) sulfites ajoutés ni intrants, dans un contexte socioculturel animé par des problématiques sanitaires et écologiques fortes, notamment liées à l'usage de pesticides et d'engrais chimiques. D'abord objets de curiosité, ces vins dits « naturels » sont peu à peu devenus un phénomène de mode qui a su séduire quelques cavistes, bars à vins et res-

1 Audrey Moutat est maître de conférences en Sciences du Langage et en Sciences de l'Information et de la Communication à l'Université de Limoges et membre du Centre de Recherches Sémiotiques (CeReS).

Recherches en communication, n48 - Article publié le 26/02/2019 
taurateurs, voire certains chefs - David Toutain, Gilles Bénard ou Gérald Passédat (pour n'en citer que quelques-uns) ont ainsi fait le pari de les ajouter à leur carte. L'attrait de ces vins serait en partie motivé par une volonté de s'extraire des standards gustatifs imposés par les $\mathrm{AOC}$ et de revenir à une production et une consommation raisonnées, en engageant des pratiques vitivinicoles émancipées des contraintes imposées par la réglementation. En laissant au raisin la possibilité d'exprimer toute la richesse de son terroir, chacun de ces vins présente une architecture sapide singulière et manifeste une palette aromatique différente des vins « conventionnels ». De telles propriétés posent alors la question de leur communication, notamment la création de nouvelles entrées lexicales, et impliquent la mise en œuvre de grilles de dégustation adaptées. Aussi, cette absence de normalisation constitue un levier créatif pour les vignerons nature qui ne cessent de jouer la carte de la transgression. Les dispositifs communicationnels engagés dans la promotion de ces vins ne s'enferment pas dans la rigueur traditionnelle des vins français : noms insolites, étiquettes atypiques ou encore commentaires de dégustation familiers... les vignerons nature s'émancipent des codes traditionnels, qu'ils jugent élitistes, afin d'engager une relation de proximité avec le consommateur.

Cet article propose ainsi d'étudier les dispositifs communicationnels mis en place lors de la promotion de ces vins, et plus particulièrement dans ce que nous identifions comme une rhétorique de la transgression.

Pour ce faire, nous adopterons une méthodologie d'analyse des études qualitatives. Dans un premier temps, il s'agira de décrire avec objectivité ce phénomène des vins naturels en nous appuyant sur des ouvrages, blogs et sites web des acteurs du milieu. Nous procéderons ainsi à une analyse de contenu de ce premier corpus : le traitement sémantique des données permettra de relever les caractéristiques principales de la viticulture nature ainsi que les éléments idéologiques sous-jacents propres à ces acteurs. Dans un second temps, nous porterons notre intérêt sur quelques-uns de ces blogs et sites web afin de déterminer leur structure énonciative, tant à l'égard du type de lexique utilisé (analyse lexicale) que dans les dispositifs énonciatifs mis en œuvre. Ce deuxième 
volet de l'étude s'inscrira plus particulièrement dans une approche sémiotique. "Science qui étudie la vie des signes au sein de la vie sociale » (De Saussure, 1971, p. 33), cette dernière ne s'intéresse pas tant aux phénomènes de signification proprement dits qu'aux dispositifs qui permettent de les faire émerger comme tels. Ainsi, nous nous attacherons précisément à identifier par induction les articulations entre les structures discursives adoptées (analyse structurale des discours) et leurs significations sous-jacentes. Ceci nous permettra de montrer les systèmes d'organisation individuels (modes d'expression et spécificités discursives employés) d'un blog en particulier, celui de la société Vins étonnants.

Focaliser notre étude sur ce blog est doublement motivé : d'une part, en raison du caractère informationnel de ce dernier. Non activiste ni polémique, ce blog assure la promotion des vins naturels selon un style explicatif, en mettant en avant les modes de production, de consommation et de conservation de ces vins. D'autre part, Vins étonnants est une société locale avec laquelle nous avons pu entamer des échanges et quelques enquêtes préliminaires à notre projet d'étude des vins naturels ${ }^{2}$.

Les autres blogs et sites web consacrés à la viticulture nature ont été sélectionnés selon différents critères : (i) leur pertinence par rapport à nos objectifs de l'analyse ; (ii) leur cohérence, autrement dit leur « caractère commun » (Greimas, 1966, p. 93-94) qui garantit l'homogénéité du corpus ; (iii) leur exploitabilité en vertu de laquelle ces blogs et sites web présentent des différences structurantes, vecteurs de signification et (iv) leur e-réputation, c'est-àdire leur positionnement dans les pages de résultats des moteurs de recherche (en l'occurrence Google).

Ainsi, en croisant ces différents critères, nous avons retenu ces sept sites web et blogs les plus populaires pour cette première analyse :

- les sites marchands : "La Bouteille », spécialisé en vins naturels et biodynamiques, "Carnet de vins » et « Les

2 En effet, cette présente étude constitue un travail préparatoire qui s'inscrit au sein d'un programme de recherche régional plus vaste consacré aux discours promotionnels des vins nature. 
Zincorruptibles » qui commercialisent des vins bio, biodynamiques et sans sulfites ajoutés ;

- le blog de «Vins étonnants », qui publie des articles réguliers sur des vins bio ou naturels, producteurs, cépages et les possibles accords mets/vin bio ou nature ;

- le site « More than organic », qui propose d'aller au-delà du biologique, ou encore celui du « Domaine de Crève-Cœur » qui ne qualifie pas explicitement son vin de naturel mais préfère utiliser les adjectifs « original » et « authentique ».

- le site «Salon des débouchées des vignerons très natures" qui délivre, selon un militantisme affirmé, des informations sur ce qu'est le vin nature, les salons qui lui sont consacrés et autres actualités.

- le site marchand « Les peaux de vins », qui commercialise de manière très engagée des vins naturels.

À ces premiers éléments de corpus s'ajoutent quelques blogs et sites web de producteurs de vins naturels promus dans ces sites ou dans les ouvrages écrits par les acteurs du domaine.

\section{Le vin naturel, entre textes et forme(s) de vie}

Les discours des vignerons nature sont fortement déterminés par leur engagement éthique et une idéologie axée sur le terroir et le respect de la nature. Sur la page d'accueil de son site ${ }^{3}$, Alexandre Bain, vigneron à Traicy-sur-Loire, expose clairement sa philosophie : « Notre idéal est de produire des vins authentiques sans nuire aux hommes, à la terre ou à l'environnement ». Cette idéologie, en faveur de pratiques agricoles raisonnées, relève de ce qu'on pourrait appeler une « forme de vie » qui, selon la pensée d'Agamben, est

une vie - la vie humaine - dans laquelle tous les modes, les actes et les processus du vivre ne sont jamais simplement des faits, mais toujours et avant tout des possibilités de vie, toujours et avant

3 http://www.domaine-alexandre-bain.com (consulté le 29/08/2018 - Actuellement indisponible). 
tout des puissances. Tout comportement et toute forme du vivre humain ne sont jamais prescrits par une vocation biologique spécifique, ni assignés par une nécessité quelconque, mais, bien qu'habituels, répétés et socialement obligatoires, ils conservent toujours le caractère d'une possibilité, autrement dit, ils mettent toujours en jeu le vivre même [...] Cela constitue d'emblée la forme-de-vie comme vie politique (Agamben, 1993, p. 13-14).

Chez Agamben, la forme-de-vie repose ainsi sur une indistinction entre les sphères privée et politique. La vie nue constitue le point d'ancrage du pouvoir et la condition même de son exercice. Toute action engagée par le sujet s'accompagne alors d'une modification de soi. " Raisons de vivre » et « façons d'agir », les formes de vie relèvent, selon Marielle Macé (2016), d'un engagement pour idée de la vie que l'on défend ou que l'on condamne et renvoient à " singularités partageables » et des "idées de vie » auxquelles on accorde une valeur dans la vie individuelle et collective. Cette idéologie s'intègre ici dans un mouvement de contestation et de mobilisation plus large : le food activism où les actions collectives de producteurs et de consommateurs se constituent en mouvement social. Nourriture et vin deviennent un élément fédérateur de mouvements politiques et sociaux pour une agriculture et une économie alternatives au système alimentaire « conventionnel ». En rupture avec la notion de typicité imposée par les AOC, cette idéologie est parfois jugée radicale et intégriste. À cet égard, le journaliste à La Revue du Vin de France Antoine Gerbelle loue « la philosophie et le travail de certains producteurs, qui ont une vraie volonté de revenir au véritable goût du raisin » mais déplore que, " souvent, le vin naturel résulte d'une idéologie anticapitaliste, anti-grande distribution, proche de l'extrême gauche $»^{4}$. On pourrait ainsi définir cette dichotomie comme deux micro-formes de vie émanant d'une forme de vie «nature » unique.

La première est raisonnée et implique un retour à une culture à l'ancienne, en s'appuyant sur des connaissances acquises ou en

4 Les vins naturels : au-delà du bio, Le Nouvel Économiste. Disponible en ligne : https://www.lenouveleconomiste.fr/dossier-art-de-vivre/les-vins-naturels-au-deladu-bio-16659/. 
expérimentation, de la vigne et du vin, tout en ayant conscience de ses limites.

Beaucoup plus radicale, la seconde apparaît comme un modèle d'insurrection qui adopte une communication plus offensive. Antonin Iommi-Amunategui (2015) l'assimile à une contreculture dans l'agriculture, initiée par des hédonistes en quête de la vérité du plaisir. Autoproclamés " pirates du vin », ces vignerons nature se considèrent comme des rebelles, à l'image du blogueur Olif qui se définit comme un « terroiriste hédoniste naturiste jurassique $»^{5}$, ou encore d'Olivier Cousin, « rebelle d'Anjou », qui se qualifie d' ' insoumis » (Iommi-Amunategui, 2015, p. 11). Fondée sur le principe des utopies pirates, cette forme de vie dissidente et militantiste s'apparente, selon Iommi-Amunategui, à une TAZ (temporary autonomous zone). Empruntée à l'écrivain politique et poète américain Hakim Bey, cette dénomination caractérise un esprit de révolte, élevé contre la loi du marché et l'hyperconsommation. Elle se traduit en l'occurrence par des valeurs de liberté, de festivité et de solidarité, " où les rapports entre individus sont différents, déhiérarchisés » (Iommi-Amunategui, 2015, p. 4) et authentiques. Elle dénonce également un paradoxe fondamental, entre la reconnaissance du vin industriel dont les intrants et autres composants exogènes ne sont pas renseignés sur l'étiquette et l'absence de légitimation du vin naturel qui n'use pourtant d'aucune levure exogène ni de sulfites (ou alors en quantités infimes lorsque cela s'avère nécessaire).

De cette forme de vie découle un réseau de significations qui, organisé en niveaux, détermine les éléments structurants de l'éthique et de la philosophie des vins naturels.

Ainsi, cette forme de vie engage en premier lieu des pratiques viti-vinicoles spécifiques refusant tout usage d'engrais et de pesticides chimiques ou encore de désherbants. "Non interventionnistes ", les vignerons de vins nature n'ajoutent aucun additif à leurs vins ; les seules manipulations restant la gestion de l'oxygène et de l'acidité volatiles des cuves et barriques. Les vendanges sont strictement manuelles, avec tri avant encuvage. Aucun filtrage ou

5 http://www.leblogdolif.com. 
collage n'est autorisé et le matériel doit être strictement nettoyé avant manipulation des moûts et du vin (cela afin d'éviter toute contamination bactérienne).

Ces pratiques font du vin naturel un objet sensible inédit doté de caractéristiques organoleptiques singulières : ses propriétés gustatives sont celles que lui ont transmis les raisins et ses saveurs relèvent de son terroir d'origine. La bouche présente une structure souple et des arômes de fruits frais et croquants, notamment grâce à la macération carbonique ${ }^{6}$. Quant à la robe, elle s'avère plutôt trouble et mate, avec quelques dépôts (en raison de l'absence de collage et de filtrage).

Ces propriétés organoleptiques singulières engagent de nouvelles pratiques de dégustation et requièrent des grilles d'analyse adaptées : le vin naturel pouvant contenir des résidus de $\mathrm{CO}_{2}$ issus de la fermentation, l'ouverture de la bouteille ou sa mise en carafe peuvent s'avérer nécessaires plusieurs heures avant la dégustation afin d'éliminer ces résidus et toute odeur de réduction (et cela de manière plus fréquente qu'avec un vin conventionnel). De plus, il requiert une conservation selon une bonne hygrométrie et à une température n'excédant pas les $15^{\circ}$.

Bien que le terme « vin naturel » soit déjà usité dans les écrits spécialisés du XVIII ${ }^{\mathrm{e}}$ siècle $^{7}$, dans un contexte où les pratiques frauduleuses commencent à se développer, il ne présente toujours pas de contenu stabilisé et n'entre dans aucun cadre réglementaire. Affranchi des réseaux de distribution, il est rejeté des AOC/AOP, dont il ne respecte pas le cahier des charges, et sa mention à titre commercial est illégale dans de nombreux pays.

Face à ce vide juridique, 1'Association des Vins Naturels $(\mathrm{AVN})$ a proposé sa propre définition :

6 Technique consistant à mettre les raisins entiers dans une cuve saturée en $\mathrm{CO}_{2}$ afin de permettre une fermentation intense.

7 Dans le cadre de nos recherches terminologiques sur le vin naturel (Colloque Les Rencontres du Clos Vougeot 2018), les dictionnaires, textes scientifiques et philosophiques anciens consultés ont mis en évidence que la désignation « vin naturel » remonte au moins au $\mathrm{XVI}^{\mathrm{e}}$ siècle. Cependant, à cette époque, elle s'avère être un simple pléonasme désignant un vin issu de la nature. C'est au XVIII ${ }^{\mathrm{e}}$ siècle que cette désignation acquiert une signification axiologique positive qui la distingue des vins coupés et altérés. 
1. un vin dont les raisins sont issus de l'agriculture biologique ou biodynamique, 2 . un vin qui est vinifié et mis en bouteille sans aucun intrant, ni additif (site de l'AVN).

Ce flou terminologique constitue un levier créatif pour les acteurs des vins nature qui usent des dispositifs communicationnels numériques (réseaux sociaux, sites web et blogs) en s'émancipant des conventions communicationnelles propres à la critique œnologique. À cet égard, on observe deux stratégies de branding différente, propre à chaque micro-forme de vie : la première est sobre, informationnelle et explicative et met en avant les modes de production des vins, respectueux des valeurs qui animent les vignerons (La Bouteille, Carnet de vins, Vins étonnants, More than organic). La seconde est transgressive et dissidente, fondant ses discours sur une critique des vins conventionnels avec un ton parfois accusateur et un esprit de rébellion (Salon des débouchées des vignerons très natures, Les Zincorruptibles, Les peaux de vins).
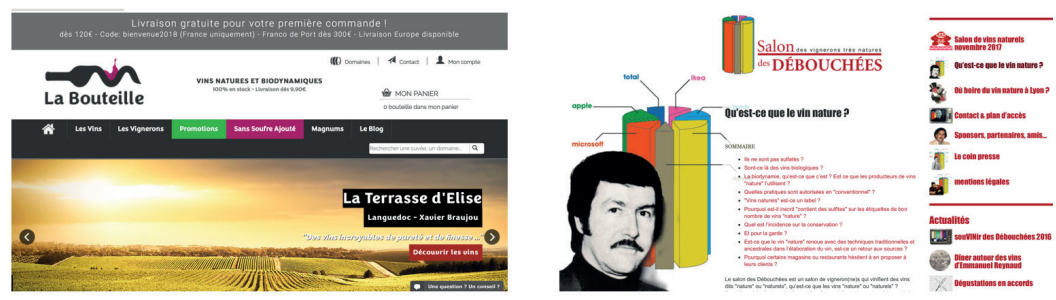

Figure 1. Exemples des deux stratégies de branding (raisonnée à gauche, transgressive à droite)

En s'adaptant au support numérique et plus particulièrement au genre du blog, ces discours mettent également en tension deux genres textuels et discursifs distincts : le blog et le commentaire de dégustation.

8 Si les vins naturels sont nécessairement produits à partir de raisins issus de l'agriculture biologique, ils se distinguent néanmoins des vins biologiques. En effet, ces derniers sont manipulés chimiquement et technologiquement. Un vin naturel ne contient pas plus de $10 \mathrm{mg} / \mathrm{L}$ de soufre pour le rouge et pas plus de $25 \mathrm{mg} / \mathrm{L}$ pour le blanc. 

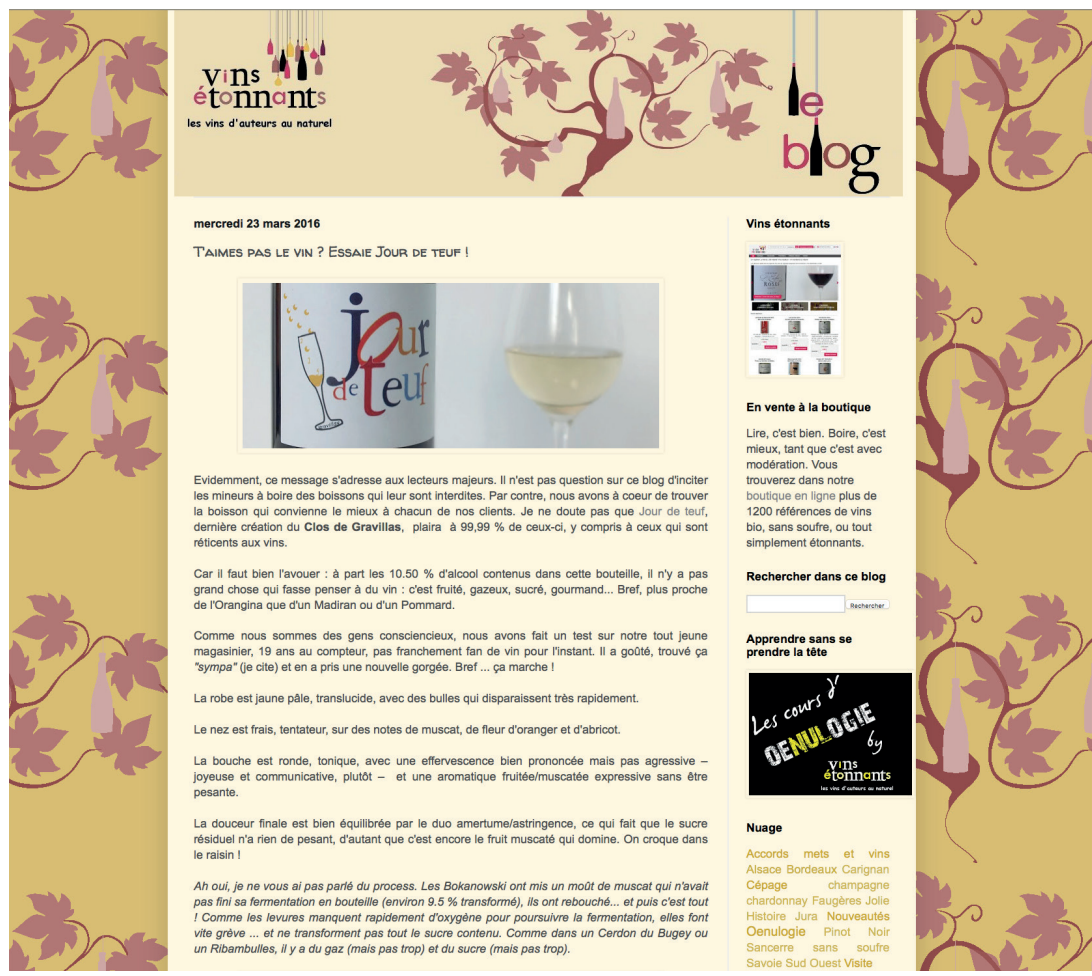

Vins étonnants

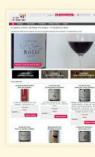

En vente à la boutique
Lire, c'est bien. Boire,
mieux, tant que c'est av

Lire, c'est bien. Boire, c'est
mieux, tant que c'est avec
modération. Vous modération. Vous
trouverez dans notre

trouverez dans notre
boutlique en ligne plus de 1200 références de vins blo, sans soufre, ou tout simplement êtonnants.
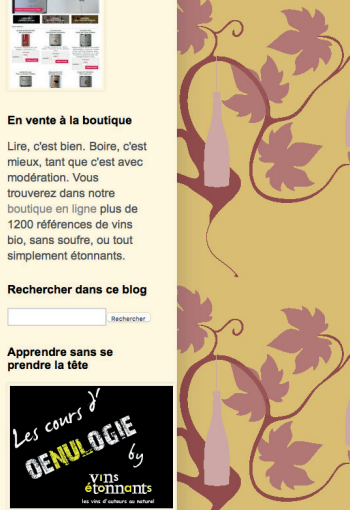

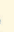
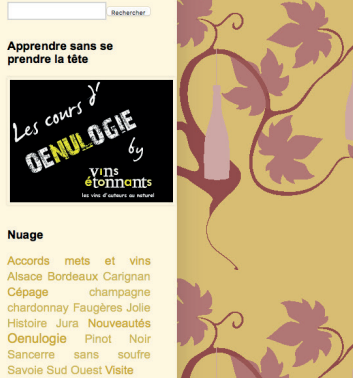

Figure 2. Exemple de billet sur le blog de Vins étonnants

Quant au caractère atypique de ces vins et à leurs propriétés organoleptiques inédites, ils ont fait émerger de nouvelles terminologies dans les lexiques de dégustation : " Juteux », " caillouteux », " salin », « sucraillon », " frizzant », « solaire », etc., tels sont les nouveaux descripteurs que nous avons pu recenser dans notre corpus.

La forme de vie des vins naturels met ainsi en jeu le réseau de plans de pertinence suivant : 


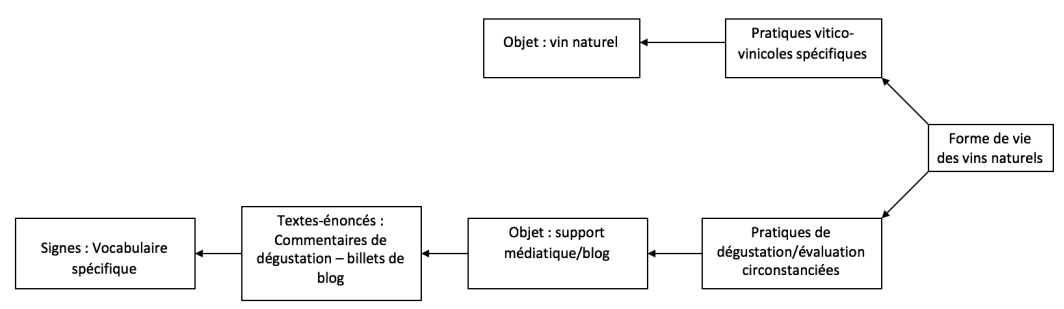

Figure 3. Niveaux de pertinence sémiotique (Fontanille, 2007)

Focalisons-nous à présent sur ce nouveau genre de commentaire afin de dégager les structures discursives à l'œuvre dans les dispositifs communicationnels sur les vins naturels.

\section{Discours sur les vins naturels}

Afin de mener à bien cette entreprise, nous avons pris pour objet d'étude le blog http://vins-etonnants.blogspot.com, lié au site web de la société Vins étonnants (https://www.vins-etonnants. com), dirigée par Éric Reppert.

\subsection{Présentation du corpus}

« Caviste sans magasin », Éric Reppert assure la promotion et la vente de plus de 500 références en vins bios et naturels sur son site web. Son concept est de proposer des « vins d'auteurs au naturel », autrement dit de faire découvrir des vins provenant de cépages rares, aux assemblages inédits ou terroirs méconnus et vinifiés selon des techniques atypiques. Créé en 2012, le blog Vins étonnants est animé par Éric Bernardin qui publie quotidiennement un article sur un vin, un producteur, un cépage, un accord met/vin mais, surtout, il engage un genre textuel nouveau : le web-commentaire de dégustation. 


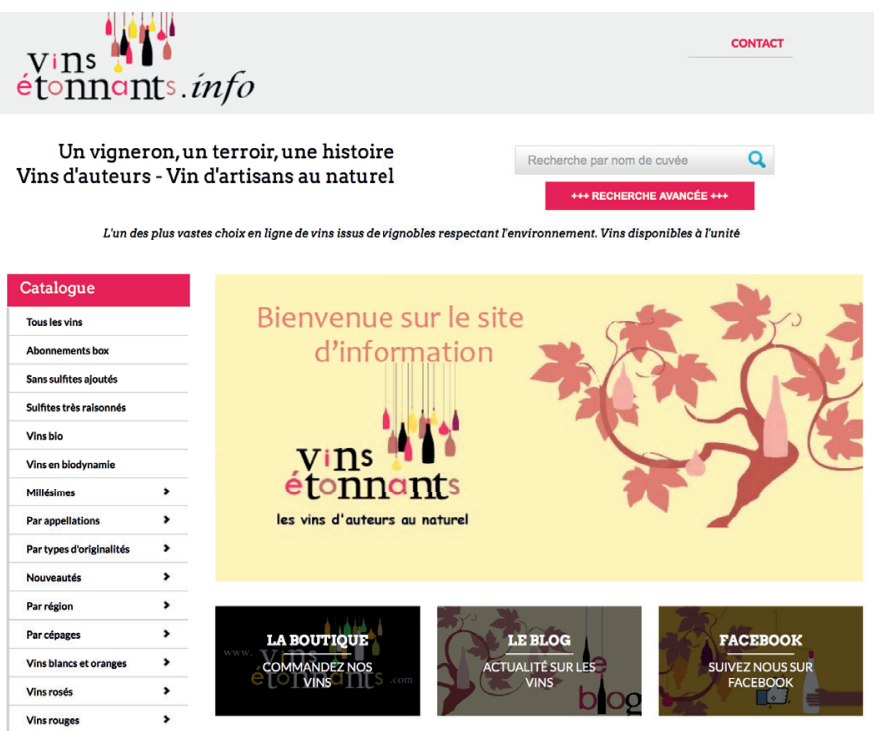

Figure 4. Page d'accueil du site Vins étonnants

\subsection{Le web-commentaire, un genre renouvelé}

« De même que les normes sociales constituent le fond qui permet de comprendre les activités individuelles, l'étude du genre revêt son plus grand intérêt quand elle permet de percevoir les singularités des textes » (Rastier, 2001). En effet, nous avons dit précédemment que si ces posts (ou billets) relevaient fondamentalement du genre du blog, ils empruntent cependant aux commentaires de dégustation, en proposant une description analytique des vins naturels. Dès lors, nous avons affaire à un genre textuel hybride, conforme à une forme de vie spécifique et dont il convient d'en déterminer les principes. Pour ce faire, nous convoquerons le principe de généricité (Couégnas, 2013).

Le gradient de généricité incarne une double tension graduelle entre des prescriptions et des normes génériques d'une part, le style et l'œuvre de l'autre. Partant d'un socle générique, cette tension déploie différents degrés : des modèles génériques et des combinaisons de modèles, aboutissant au style qui préside à la singularité de l'œuvre. Le gradient de généricité fonctionne comme 
un arbre générique pouvant se lire comme une suite de spécifications successives ou comme une remontée de déterminations en déterminations.

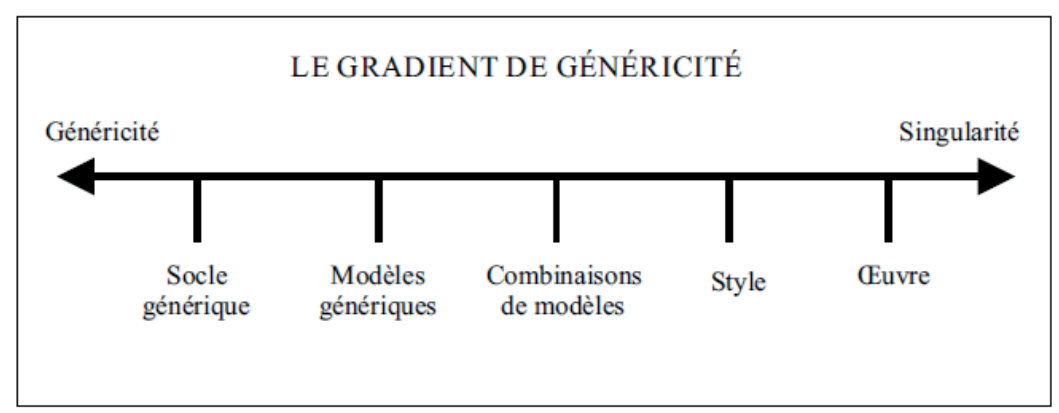

Figure 5. Le gradient de généricité

Le socle générique correspond aux sélections et spécifications des composantes textuelles définitoires du genre. Ce socle se trouve ensuite incarné dans des modèles génériques organisés en combinaisons élémentaires pouvant ensuite donner lieu à des possibilités combinatoires logiques de modèles. Le style se caractérise, quant à lui, par une appropriation des contraintes précédentes : il peut les maintenir, les ajuster ou bien les modifier totalement ou partiellement. C'est à partir de ce style que se définit la singularité de l'œuvre.

Notre corpus de billets du blog de Vins étonnants met en évidence des emprunts à plusieurs genres : l'écriture numérique, le récit autobiographique propre au blog et le commentaire de dégustation. L'ensemble constitue un genre nouveau que l'on pourrait qualifier de blog œnologique, et plus particulièrement de web-commentaire quotidien de dégustation. Son socle générique, identifiable à partir des différentes composantes textuelles (Rastier, 2001) de ces textes, se trouve tout d'abord déterminé par des spécificités propres au blog. 

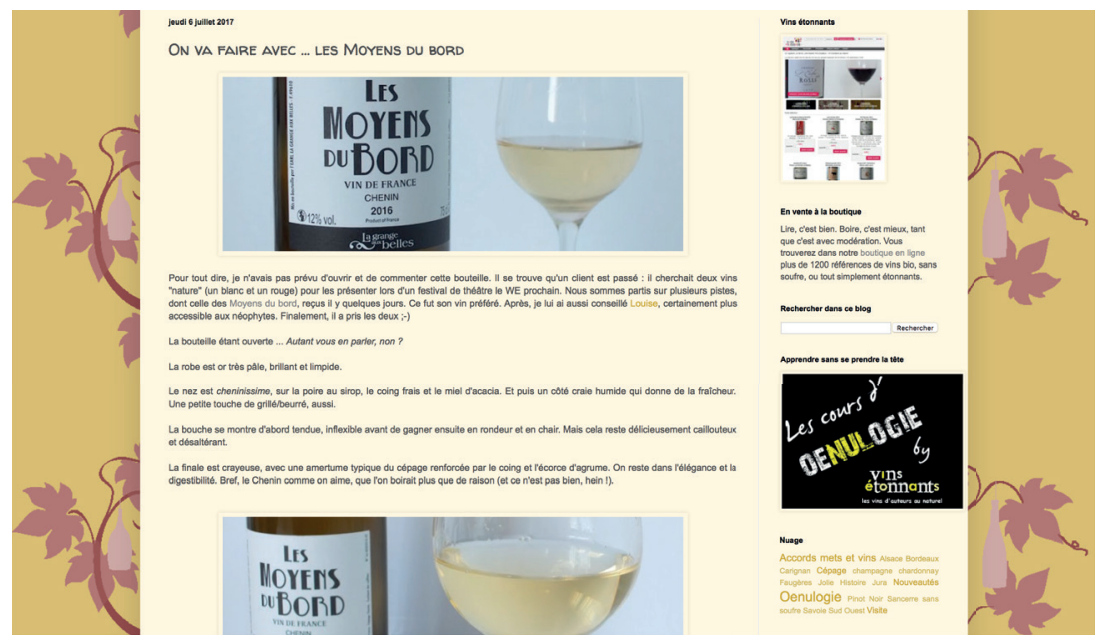

Figure 6. Web-commentaire du vin Les moyens du bord

En effet, le billet se caractérise par sa taille relativement modérée (pas plus de 1600 caractères) et une structure en entonnoir : l'introduction contextualise l'article par une brève histoire ou une anecdote, suivie d'un avis argumenté faisant office de note de dégustation, puis d'une conclusion synthétique, offrant parfois une ouverture. Quant au blog lui-même, il suit une organisation anti-chronologique où le billet le plus récent apparaît sur la première page tandis que les plus anciens s'inscrivent dans la profondeur du site. Le vocabulaire convoqué est familier ("à donf" ", « cartonner », « niaque », « too much », « une p... de fraîcheur »...) et emprunte au langage parlé : «C'est fou, ça ! [...] je ne suis déjà plus à la page... », « Que dire de plus ? J'a-dore ! », " Ça nous fait vraiment plaisir de rendre service :-) »... 

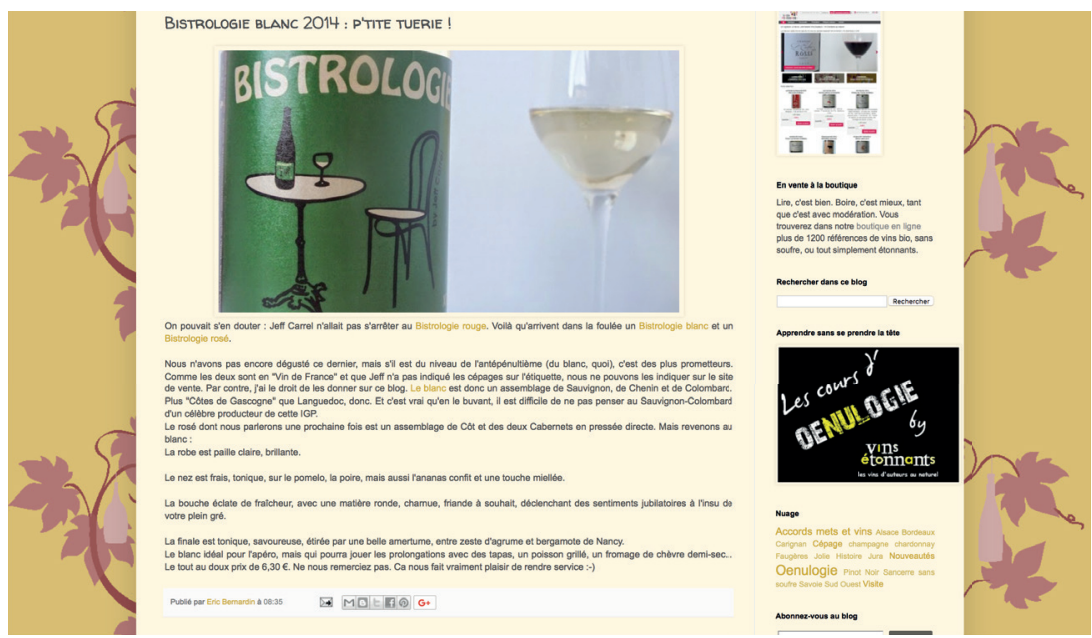

Figure 7. Web-commentaire du vin Bistrologie

On retrouve certaines caractéristiques propres à la composante médiatique du blog : smileys, images, liens cliquables et boutons sociaux, lesquels favorisent une communication interpersonnelle entre le dégustateur-blogueur et ses lecteurs. Cette connivence se manifeste également à travers des effets prosodiques (interjections et intonations) qui exercent une fonction d'interpellation : " "Pas de soirée dans K-Pot!" vous direz-vous une fois que vous aurez découvert le millésime 2014 du vin sans sulfites ajoutés du Clos Troteligotte », "La bouteille étant ouverte... Autant vous en parler, non? », « Mais le contenu est vraiment É-PA-TANT! ».

D'autre part, le billet présente certaines caractéristiques relevant du commentaire de dégustation. Il se réfère au vin et à ses propriétés organoleptiques en convoquant une terminologie articulée par deux ensembles : un lexique « qualitatif» composé de descripteurs chromatiques, aromatiques et sapides du vin et un lexique « structurel » qui détermine l'armature visuelle, olfactive et gustative de ces propriétés (Moutat, 2015). Ce second lexique se compose de descripteurs pouvant relever de domaines sémantiques différents de celui du vin tels que les propriétés physico-anthropologiques (" élégant », " fin », " charnu », " maigre »), psychoanthropologiques ( agressif », « discret », « franc », « sincère ») 
ou sociales (« noble », « riche », « puissant») de l'être humain, la géométrie ou la plastique (« rond », « cônique », « ample », «plat»).

Tel le commentaire classique, la note de dégustation du billet possède une organisation tripartie correspondant au découpage séquentiel de l'évaluation du vin en robe, nez et bouche. Ce découpage ne relève pas du déploiement des propriétés organoleptiques du vin mais d'une organisation rationnelle des perceptions du dégustateur. La composition rythmique du billet de dégustation se présente quant à elle comme un condensé descriptif de structures organoleptiques où sont accumulés, voire apposés les uns aux autres, les descripteurs : « La bouche est pure, fraîche, d'une bonne ampleur, avec des bulles bien présentes mais pas du tout agressives, et une matière ronde, fruitée, bien mûre »; «Le nez est bien mûr tout en restant aérien, sur des notes de pâte d'amande, de miel, de pomme tapée et de mirabelle séchée (oui, ça existe dans le Sud-Ouest) ».

Ce condensé descriptif place le lecteur dans une situation semblable à celle du dégustateur : comme lui, il se trouve en présence d'une multiplicité d'éléments à saisir, à la différence près qu'il s'agit ici de percevoir non pas des qualités sensibles mais des structures discursives. Cette immersion se trouve renforcée par l'usage d'expressions prépositionnelles spatialisantes telles que « sur des notes fruitées », " tirer sur », « marqué par », « aller sur », des syntagmes « avec des notes de... », « touche », ou encore des verbes « évoquer », « tendre vers », « aller vers ». Ces derniers ne procèdent pas à une classification des propriétés du vin mais proposent « plutôt de singulariser des accès, de traiter les sensations comme des devenirs de l'expérience du buveur, plutôt que d'en faire des constats catégoriels » (Cadiot, 2010, p. 49).

À partir de ce socle générique, Éric Bernardin combine les modèles propres au blog et au commentaire œnologique. En adoptant une syntaxe complète comme dans le billet de blog, le web-commentaire de dégustation refuse au commentaire stan- 
dard sa brièveté 9 . Sur le plan dialogique, il présente un caractère hybride, oscillant entre la subjectivité du blogueur et la relative objectivité du dégustateur qui évalue le vin de manière critique et technique.

\begin{tabular}{|c|c|}
\hline \multicolumn{2}{|c|}{ Socle générique } \\
\hline Blog & Commentaire de dégustation \\
\hline Taille modérée (distribution) & $\begin{array}{l}\text { Vin et propriété organoleptiques } \\
\text { (thématique) }\end{array}$ \\
\hline Scansion (distributionnel/dialectique) & $\begin{array}{l}\text { Lexique quantitatif et structurel } \\
\text { (thématique) }\end{array}$ \\
\hline Anti-chronologique (dialectique) & Vocabulaire imagé (thématique) \\
\hline Vocabulaire familier/oralité (dialogique) & $\begin{array}{l}\text { Organisation tripartie (distributionnelle/ } \\
\text { dialectique) }\end{array}$ \\
\hline $\begin{array}{l}\text { Texte, images, boutons sociaux, smileys } \\
\text { (médiatique) }\end{array}$ & Cumul de descripteurs (rythmique) \\
\hline \multirow[t]{3}{*}{ Interjection/intonation (prosodie) } & $\begin{array}{l}\text { Transformation de l'univers du dégusta- } \\
\text { teur (dialogique) }\end{array}$ \\
\hline & Spatialisation (dialogique) \\
\hline & $\begin{array}{l}\text { Singularisation des accès aux propriétés } \\
\text { organoleptiques (dialogique) }\end{array}$ \\
\hline
\end{tabular}

Figure 8. Socle générique du web-commentaire de dégustation

En outre, ce genre singulier présente une composante monothématique, celle de la relation du vin (et notamment d'un vin atypique) avec son dégustateur. Mais sa véritable particularité se situe dans le corrélat des composantes distributionnelle et tactique : le web-commentaire se présente comme un condensé figuratif qui procède à la synthèse de la pratique itérative de la dégustation et engage en retour une pratique interprétative similaire à cette séquence de dégustation. En effet, au contact du vin, le dégustateur a affaire à une diversité sensible qui nécessite une itération de ses gestes et actes perceptifs afin d'en déceler les subtilités. Dès lors, deux aspectualités concurrentes se mettent en place : le procès d'exfoliation des qualités organoleptiques du vin qui est de l'ordre

9 En effet, les commentaires de dégustation publiés dans la presse œnologique adoptent généralement une syntaxe minimale, le plus souvent averbale et sans déterminant. 
du [continuatif - duratif - progressif] et la réitération des actes perceptifs du dégustateur, caractéristique du procès de dégustation [itératif - discontinuatif - pause].

La mise en récit de cette séquence de dégustation repose ainsi sur une homogénéisation des saisies perceptives du dégustateur dans un énoncé dense et synthétique. Face à un tel condensé descriptif, le travail interprétatif engagé par le lecteur nécessite une relecture des fragments textuels et une réévaluation permanente de ses inférences interprétatives ; lesquelles font écho à l'itération des gestes perceptifs du dégustateur.

Socle générique

- Commentaire étendu ; le billet n'emprunte pas sa brièveté. Le commentaire classique se combine avec le billet de blog (distributionnel)

- Effacement de l'univers de référence du blogueur au profit d'un univers de référence plus technique (dialogique)

- Vin, terroir, propriétés organoleptiques, accords mets/vins (monothématique)

- Corrélat composantes distributionnelle et tactique

Figure 9. Modèles du web-commentaire de dégustation

C'est à ce niveau que se manifestent les caractéristiques proprement stylistiques du blog Vins étonnants. Sur le plan distributionnel, le morcellement de l'information est aléatoire. Les billets ne s'inscrivent pas forcément dans la continuité. Si le continuum est d'ordre thématique ${ }^{10}$, il n'existe pas forcément de lien de cause à effet ni de continuum temporel entre les billets si bien que, l'expression n'étant pas morcelée, les billets peuvent être lus de manière aléatoire.

En outre, ces web-commentaires offrent la particularité de convoquer le lexique qualificatif et structurel en fonction d'attribut et non en fonction épithète, comme cela se fait ordinairement dans le commentaire de dégustation. Le niveau dialogique se caractérise par un récurrent jeu d'allers et retours entre le mode personnel et le mode impersonnel.

10 Il s'agira toujours du vin, de ses qualités organoleptiques et de ses accords possibles avec les mets. 

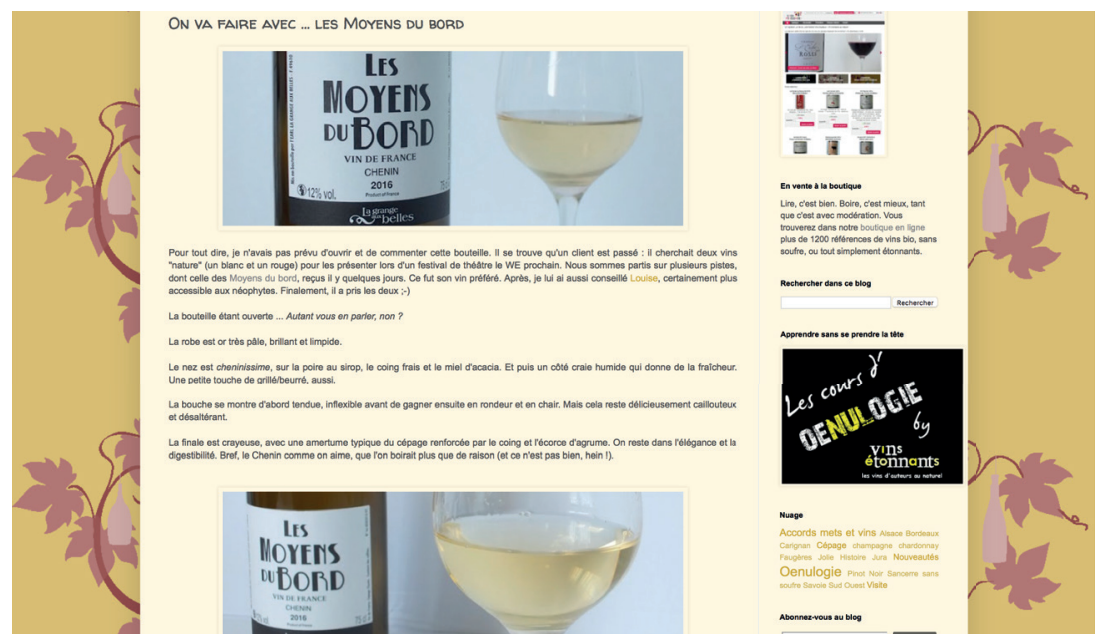

Figure 10. Modes personnel et impersonnel du web-commentaire

Dans ce billet, le blogueur interpelle directement le lecteur par une anecdote personnelle à partir de laquelle il amorce le sujet du jour. Dans l'introduction, il s'exprime donc à la première personne du singulier avant de s'adresser directement à son lecteur par une question rhétorique qui crée une bascule vers le discours impersonnel du commentaire. À la subjectivité marquée de l'anecdote s'oppose un discours technique et objectif. Ce n'est qu'au terme de cette critique œnologique que le discours se veut plus personnel et familier avec l'emploi d'un « on » collectif qui renvoie à la fois au blogueur mais également à ses lecteurs.

Ces jeux entre discours personnel et impersonnel opèrent variablement au fil des billets. Le blogueur peut tantôt engager sa propre subjectivité en qualité d'individu, tantôt s'exprimer au nom d'un « nous » collectif qui engage cette fois-ci sa responsabilité professionnelle. Tel est le cas des exemples suivants : «Par contre, nous avons à cœur de trouver la boisson qui convienne le mieux à chacun de nos clients", "Comme nous sommes des gens consciencieux, nous avons fait un test sur notre tout jeune magasinier, 19 ans au compteur, pas franchement fan de vin pour l'instant ». 
Cependant, il peut également faire usage du « nous » afin de s'intégrer à la collectivité des énonciataires, amateurs de vins naturels. Dans ce second cas, le blogueur n'est plus animateur de la société Vins étonnants mais un passionné, leader d'opinion d'une communauté à laquelle il se revendique d'appartenir. Ainsi le style du blog Vins étonnants manifeste-t-il cette tension entre un « nous » statutaire où la subjectivité et la personnalité du blogueur se trouvent complètement effacées, et un « nous » communautaire qui, au contraire, affirme cette personnalité dans un groupe animé par une passion commune.

Sur le plan dialectique, l'évaluation des qualités gustatives en attaque/évolution/finale se trouve remodelée en une synthèse générique des deux premières et une focalisation sur l'impression finale laissée en bouche.

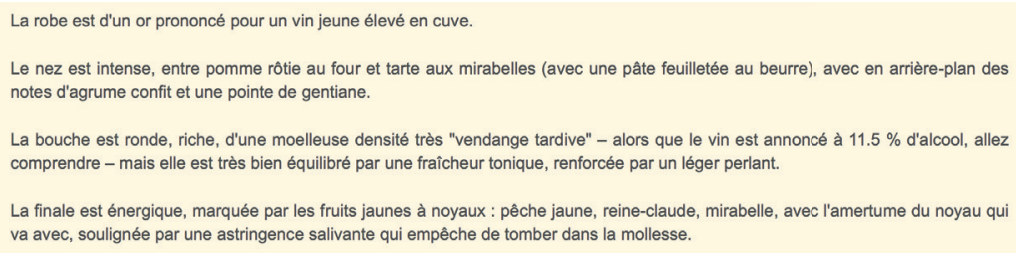

\section{Figure 11. Une évaluation des qualités gustatives renouvelée}

Enfin, sur le plan thématique, le lexique aromatique se trouve enrichi en raison des qualités organoleptiques spécifiquement marquées des vins naturels. De nouvelles entrées intègrent ainsi des catégories aromatiques peu convoquées dans l'évaluation positive des vins conventionnels : 1 . Le minéral est exprimé en termes de " pierrosité », " caillouteux », " schiste », " craie humide », « crayeux », « roche de Leucate », « kimméridgien » renvoyant au caractère du terroir particulièrement affirmé dans le vin nature. 2. Ces arômes ainsi décrits entrent en complémentarité avec les arômes marins ("salin ») qui apportent un appui à cette minéralité. 3. Les descripteurs du végétal « feuille froissée », « terre retournée ", " herbe froissée " renvoient aux arômes tertiaires développés durant l'élevage du vin. Ces arômes résultent d'une modification de leurs composants sous l'action de l'oxygène. 4. Ils 
se trouvent parfois accompagnés par les arômes empyreumatiques (« encens », " fumée »). 5. Le lacté s'exprime à travers les arômes de « saveurs yaourtières » ou " lactés », issus de la fermentation malolactique où l'acide malique du raisin se trouve transformé en acide lactique.

Les descripteurs utilisés dans notre corpus affirment les propriétés naturelles, authentiques, parfois rustiques, de ces vins très proches de leurs origines. Les cépages se trouvent particulièrement expressifs comme en attestent les descripteurs « cheninesque » ou encore « pinoter».

Au niveau de la texture en bouche, le caractère du fruit semble prendre de l'ampleur, comme en atteste l'emploi fréquent du descripteur « juteux ». Si le « perlant» peut parfois subsister, cette caractéristique est très souvent minimisée et considérée comme non nuisible à l'ensemble de la structure du vin.

Quant aux descriptions des propriétés visuelles, elles font appel à une terminologie considérée comme dépréciative sur les échelles évaluatives des vins conventionnels : « translucide », par exemple, est un descripteur typiquement employé dans l'évaluation de la robe. Il s'oppose à « transparent », utilisé pour décrire les qualités d'un vin conventionnel mais se distingue également de «trouble » qui caractérise une robe altérée. "Translucide » permet ainsi de décrire une robe sans défaut, conforme aux critères d'évaluation des vins naturels. Elle ne peut être transparente dans la mesure où, au cours de la vinification, il n'est fait usage d'aucun blanc d'œuf pour coller les impuretés et autres particules en suspension produites au cours de l'élevage.

Bien que « violacé » et «pâle » désignent une robe dont la couleur n'est pas véritablement marquée, ils ne sont pas considérés comme des qualificatifs dépréciatifs, à la différence des grilles analytiques des vins conventionnels. Au contraire, ils certifient l'origine et la qualité d'un produit naturel dont la robe présente une teinte subtile.

Les particularités organoleptiques des vins naturels imposent ainsi de nouvelles échelles évaluatives et une réévaluation de la terminologie œnologique. De ce fait, si un défaut jugé préjudiciable à un vin conventionnel est décelé, il sera ici considéré comme l'une 
des « tranches de vie » d'un vin au caractère « vitaliste ». Lorsqu'il est mentionné dans le web-commentaire, ce « défaut » se trouve immédiatement minimisé par des conseils de dégustation invitant à la patience (conservation en cave ou carafage). Le blogueur use alors de structures euphémisantes ou de qualificatifs venant rééquilibrer le jugement qualitatif initial afin de prévenir toutes critiques de ses détracteurs :

« La finale légèrement accrocheuse trahit la grande jeunesse de ce vin. Je pense qu'elle gagnera en douceur dans les mois qui viennent. Elle offre déjà du plaisir, avec toujours cette aromatique de fruit frais, de fleur et de poivre fumé. »

« La bouche est ronde, tonique, avec une effervescence bien prononcée mais pas agressive - joyeuse et communicative, plutôt - et une aromatique fruitée/muscatée expressive sans être pesante. » «Le nez est d'abord bien réduit. Il est donc conseillé de carafer le vin ou de l'ouvrir la veille en en prélevant un verre. Le nez est alors beaucoup plus avenant : pivoine, fruits rouges (fraise confite, framboise fraîche), noyau de cerise, tabac, épices. »

\begin{tabular}{|l|}
\hline \multicolumn{1}{|c|}{ Style } \\
\hline - Lien de cause à effet ou continuum temporel non systématique entre les billets \\
- Aller et retour entre le mode personnel et le mode impersonnel (dialogique) \\
- Focalisation sur la finale (dialectique) \\
- De nouvelles entrées lexicales et grilles évaluatives (thématique)
\end{tabular}

Figure 12. Style du web-commentaire de dégustation

Quant à la singularité du web-commentaire de dégustation, elle ne peut être définie à ce stade de notre étude et requiert un travail comparatif avec d'autres blogs consacrés aux vins naturels. Cela nous permettra alors de vérifier si ces singularités opèrent comme des micro-structures de l'expression renvoyant à d'autres types de micro-formes de vie du vin naturel. 


\section{Conclusion}

Dans ce travail, nous avons montré comment la forme de vie $\mathrm{du}$ vin naturel a pu faire émerger un nouveau genre textuel (le web-commentaire quotidien de dégustation) et avons ainsi esquissé un parcours des stratégies discursives à l'œuvre dans ces récits. Une analyse sémantique et sémiotique approfondie de ces textes demeure néanmoins nécessaire afin de déceler plus précisément les qualités sensibles des vins naturels et notamment ce qui les différencie des vins conventionnels. Cela permettra de déterminer de nouveaux profils organoleptiques dans le paysage œnologique et de mettre au jour des grilles d'analyse spécifiques. Un autre axe est également à envisager, celui de l'anormalisation de l'étiquette et de l'atypie dénominative de ces vins. Les noms de domaines et de cuvées ont ainsi laissé leur place à des noms insolites, empreints d'humour et de provocation: "Le lit' », « White is white », « Jour de teuf », " Bergecrac », " K-pot' " ou encore "You fuck my wine $» .$. Loin des représentations de domaines, châteaux ou blasons assurant la promesse d'excellence d'un produit culturel raffiné, l'étiquette du vin naturel mise sur une stratégie d'assomption (Jeanneret \& Souchier, 1999) en adoptant une typographie fantaisiste, des photographies de star du cinéma, des cases de BD ou des représentations iconiques atypiques (tête de mort, spermatozoïdes ou corps dénudés)... Ainsi envisageons-nous de poursuivre nos investigations en ce sens en mesurant l'impact de tels discours sur les consommateurs et leur pertinence avec la micro-forme de vie qui les sous-tend.

\section{Références}

Agamben, G. (1993). Moyens sans fins. Notes sur la politique. Paris: Rivages Payot. Cadiot, P. (2010). Le vin en paroles. Esquisses æenophiles. Perros-Guirec : Anagrammes. Couégnas, N. (2013). Sémiotique textuelle du genre. La généricité des albums d'enfance. Dans D. Ablali, Théories et pratiques des genres. Pratiques 157-158, 7690. Disponible à : https://journals.openedition.org/pratiques/3671. Doi : 10.4000/ pratiques.3671.

De Saussure, F. (1971). Cours de linguistique générale. Paris : Payot.

Fontanille, J. (2007). Textes, objets, situations et formes de vie. Les niveaux de pertinence du plan de l'expression dans une sémiotique des cultures. Dans D. Bertrand 
\& M. Costantini (Éd.), Transversalité du Sens. Paris : Presses universitaires de Vincennes. Disponible à : https://www.unilim.fr/pages_perso/jacques.fontanille/ textes-pdf/CSituations pratiques2004_06.pdf.

Fontanille, J. (2015). Formes de vie. Liège : Presses universitaires de Liège.

Greimas, A.-J. (1966). Sémantique structurale-Recherche de méthode. Paris : Larousse. Iommi-Amunategui, A. (2015). Manifeste pour le vin naturel. Paris : Les éditions de l'Épure - Marie Rocher.

Jeanneret Y. \& Souchier E. (1999). L'étiquette des vins : analyse d'un objet ordinaire. Communication et langages, 121 ( $3^{\mathrm{e}}$ trimestre), Dossier : L'université d'été de la communication, 72-85. Doi : https://doi.org/10.3406/colan.1999.2949.

Macé, M. (2016). Styles. Critique de nos formes de vie. Coll. NRF Essais. Paris : Gallimard.

Moutat, A. (2015). Du sensible à l'intelligible. Pour une sémiotique de la perception. Limoges : Lambert Lucas.

Rastier, F. (2001). Arts et sciences du texte. Paris : PUF.

Savard, V. (2016). Les formes de vie : une nouvelle façon d'appréhender l'individu et la société dans l'œuvre littéraire, Postures, Actes du colloque « Réfléchir les espaces critiques : consécration, lectures et politique du littéraire » 24. Disponible à : http:// revuepostures.com/fr/articles/savard-24.

Siniscalchi, V. (2015). «Food activism » en Europe : changer de pratiques, changer de paradigmes, Anthropology of food, S11. Disponible à : http://journals.openedition. org/aof/7920. 


\section{(c) (1) $(9)$}

«Attribution - Pas d'Utilisation Commerciale - Pas de Modification 4.0 International» (CC BY-NC-ND) 